\title{
Multiple Classification Algorithm Based on Graph Convolutional Neural Network for Intrusion Detection
}

Fulai Liu ( $\sim$ fulailiu@126.com )

Northeastern University https://orcid.org/0000-0001-6592-1028

Zhongyi Hu

Northeastern University

Aiyi Zhang

Northeastern University

Ruiyan Du

Northeastern University

Dongbao Qin

Northeastern University

Jialiang Xu

Northeastern University

\section{Research Article}

Keywords: Intrusion detection, Graph convolution neural network, KDD-CUP 99

Posted Date: June 3rd, 2021

DOI: https://doi.org/10.21203/rs.3.rs-515900/v1

License: (9) (i) This work is licensed under a Creative Commons Attribution 4.0 International License. Read Full License 


\title{
Multiple Classification Algorithm Based on Graph Convolutional Neural Network for Intrusion Detection
}

\author{
Fulai Liu ${ }^{1,2, *}$, Zhongyi Hu ${ }^{2, *}$, Aiyi Zhang ${ }^{2, *}$, Ruiyan Du ${ }^{1,2}$, Dongbao \\ Qin $^{2}$, Jialiang $\mathrm{Xu}^{2}$
}

Received: date / Accepted: date

\begin{abstract}
In order to improve the recognition performance of intrusion detection, a graph convolutional neural network for multiple classification intrusion detection is proposed, named as GCNID. Firstly, the detection data are preprocessed by numerical and normalized, so that the disadvantage effect of numerical differences among various features can be reduced. Then, the adjacency matrix for intrusion data in GCIND is constructed by the $k$-nearest neighbor method, which utilizes the Euclide distance between different features as evaluation criterion. Besides, the adjacency matrix and the modified detection data are set as the input of GCNID framework. Furthermore, the neighbors' information of each detection data are added into itself by the graph convolutional layer, such that more effective intrusion features can be extracted by GCNID. What's more, the parameters in the proposed method are optimized by back propagation until the network convergence. Finally, the Softmax logistic regression model is used to classify the types of intrusion. Simulation results show that the proposed method can not only improve the detection accuracy, but also classify the unknown attack types. The performance shows that the GCNID has a good ability of accuracy and generalization.
\end{abstract}

Keywords Intrusion detection · Graph convolution neural network · KDD-CUP 99

\section{Introduction}

With the rapid development of network technology, open network environment is easier to be influenced by various attacks. There are various popular network attacks such as denial of service (DoS), probing (Probe), remote to local (R2L), user to root (U2R), etc [1]. It is note worthy that the network intrusion detection becomes an effective way to prevent these attacks [2]. The network intrusion detection can actively monitor network events to identify security issues occured in the network [3]. Therefore, intrusion detection is one of key techniques for network security research [4].

In recent years, many intrusion detection methods have been proposed, such as expert system detection intrusion technology, which matches the observed data with the predefined descriptions of intrusion features [6]. It can recognize attacks in the signature database, but ineffective for detecting unknown attacks. To further improve the recognition accuracy, many successful network intrusion detection technologies based on deep learning algorithm are proposed [7], [8]. Deep learning algorithms get rid of the reliance on feature engineering and are able to intelligently identify attack features, which is benefit for identifying potential security threats [9]. An intrusion detection method is proposed for the KDD-CUP 99 dataset by using a new deep neural network (NDNN) model [10]. The attack features from network intrusion data are extracted by the NDNN model with four fully connected hidden layers. Though it achieves good results, the data information extraction is not sufficient. Recursive neural network (RNN) including the long short term memory (LSTM) and gated recurrent unit (GRU) can effectively obtain the correlation between the time series data. A network intrusion detection system is proposed based on LSTM architecture model, in which the hyperparameters are chosen by extensive experiments comparison [11]. By comparing it with other IDS classifier, experiment results show that the attacks are well detected by LSTM-based intrusion detection framework. A multiclass network intrusion detection model named CNNID is proposed based on convolutional neural networks $(\mathrm{CNN})$ to extract the structural information of intrusion data [13]. The CNNID model converts the original intrusion

\footnotetext{
${ }^{1}$ Engineer Optimization \& Smart Antenna Institute, Northeastern University at Qinhuangdao, Qinhuangdao, China.

${ }^{2}$ School of Computer Science and Engineering, Northeastern University, Shenyang, China.

*Corresponding author: Fulai Liu (fulailiu@126.com) Zhongyi Hu (zhongyi_hu@126.com) Aiyi zhang (xiaozhangaiyi@163.com). The first three authors contributed equally to this work and can be considered co-first authors.
} 
detection datasets into two-dimensional like pictures in order to meet the input requirements of neural network. The experimental results show the detection model improves the accuracy and checking rate, but the higher dimensional data may cost more overhead. To further improve the recognition performance of intrusion detection model, this paper introduces graph convolutional network (GCN) into network intrusion detection.

As an extension of classical CNN, GCN is a deep learning method of graph structure data for semi-supervised classification [14]. Because there are correlations between network intrusion data features, a graph structure of different data features can be constructed to describe the correlations [15]. This paper presents a multiple classification network intrusion detection model based on GCN, named as GCNID. Initially, the detection data are preprocessed by numerical and normalized methods to reduce the adverse effect of dimension differentiation among features. Then the adjacency matrix and graph structure of intrusion data features is given by $k$ nearest neighbor method. Finally, the adjacency matrix and network intrusion data are used as the input of GCN to identify the attack features and obtain test results of multi-class network intrusion detection. The formulation of the proposed algorithm based on GCNID is discussed in Sect.2. In Section 3, simulation results are presented to verify the performance of the proposed approach. Section 4 concludes the paper.

\section{Algorithm Formula}

The architecture of the network intrusion detection algorithm based on graph convolutional neural network is shown in Fig. 1, which is composed of the data preprocessing module, the feature learning module, and the classifier module. The graph convolutional neural network can aggregate the network intrusion data information of neighbor features. Therefore, the proposed model can achieve an excellent detection performance.

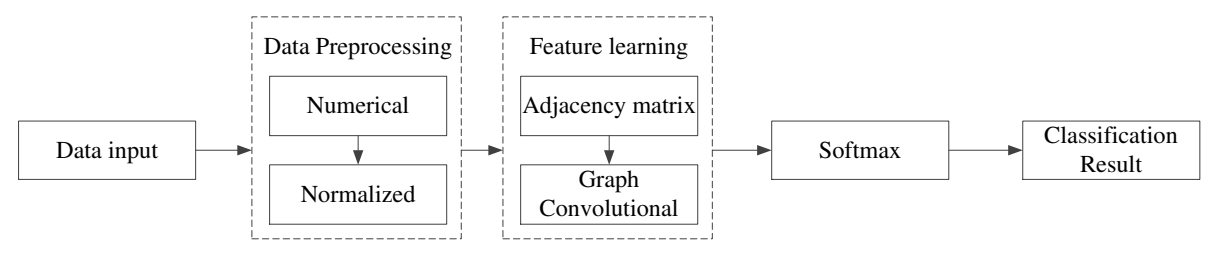

Fig. 1: GCN-based network instruction detection architecture

\subsection{Data Preprocessing}

The network intrusion data are preprocessed by numerizing text features and normalizing numerical features.

In the most of deep learning models, the data input of the neural network can use only numerical values for training and testing. The intrusion detection datasets KDD-CUP 99 contains both of text features and numerical features, so the intrusion data need to be transformed into a uniform data standard before they can be analyzed. In this subsection, a direct encoding method is used for numerizing text features. This method sorts text features based on alphabetical order, then features are encoded with $[1,2, \ldots, N]$, where $N$ represents the total number of communication protocal types (eg. "icmp" = 1, "tcp" = 2 and "udp" = 3). The encoded method dose not change the features dimension of intrusion data, so that the train and test time of detection algorithm are not increased. Furthermore, one-hot method is used to convert the labels of intrusion dataset into binary vector form (eg. "normal" $=[1,0,0,0,0]$, "DOS" $=[0,1,0,0,0]$ ), such that the labels are more suitable to train multiple classification network.

After the numerizing of text features, the features need to be normalized because the difference values among features may influence the identification accuracy. The data normalization method maps linearly all numerical features into [0,1] by using Min-Max transformation as follows

$$
x_{j}^{i}=\frac{x_{j}^{i}-x_{j}^{\min }}{x_{j}^{\max }-x_{j}^{\min }}
$$

where $x_{j}^{i}$ is the $i$ th network intrusion sample of the $j$ th feature. $x_{j}^{\min }$ and $x_{j}^{\max }$ are the minimum and maximum values of the $j$ th feature, respectively.

\subsection{Feature Learning}

The main function of the feature learning module uses GCN to automatically learn and extract useful features from the intrusion data samples. The basic structure of the intrusion feature learning with GCN is shown in Fig.2. 
The feature learning module mainly includes constructing a graph of intrusion data features, convolution operations, activation functions and optimization algorithms [16].

The values of intrusion feature can be seen as graph vertices $\boldsymbol{X}=\left\{\boldsymbol{x}_{1}, \boldsymbol{x}_{2}, \boldsymbol{x}_{3}, \ldots, \boldsymbol{x}_{n}\right\}$. Then the $k$ nearest neighbour graph method is employed to constructing the adjacency matrix $\boldsymbol{A}$ of intrusion feature. Each vertex in the intrusion feature graph is only connected its $k$ nearest neighbour features. Invoking the graph theory, an undirected graph of intrusion detection is built as $\mathscr{G}=\{\boldsymbol{X}, \boldsymbol{A}\}$, which can better represent the relationship between network intrusion data features.

In the processing of $k$ nearest neighbour graph method, the Euclidean distance between different features $D$ is expressed as

$$
D=\left(\boldsymbol{x}_{j}, \boldsymbol{x}_{n}\right)=\sqrt{\sum_{q=1}^{Q}\left(x_{j}^{q}+x_{n}^{q}\right)^{2}}
$$

where $x_{j}$ and $x_{n}$ stand for the $j$ th and $n$th intrusion feature respectively. $Q$ denotes the total data quantity. $x_{j}^{q}$ is the $q$ th samples of the $j$ th feature.

According to the Euclidean distance $D$ between different features, select $k$ features with the smallest distance for each feature as the neighbour feature nodes. Let $\mathscr{M}$ express a set of neighbour feature nodes and $\mathscr{N}$ stand for a set in which feature nodes are not directly connected. $\mathscr{M}$ and $\mathscr{N}$ are given as

$$
\begin{gathered}
\mathscr{M}=\left\{\left(\boldsymbol{x}_{j}, \boldsymbol{x}_{n}\right) \mid \boldsymbol{x}_{j} \text { and } \boldsymbol{x}_{n} \text { are neighbour feature nodes }\right\} \\
\mathscr{N}=\left\{\left(\boldsymbol{x}_{j}, \boldsymbol{x}_{n}\right) \mid \boldsymbol{x}_{j} \text { and } \boldsymbol{x}_{n} \text { are not neighbour feature nodes }\right\}
\end{gathered}
$$

where $x_{j}$ and $x_{n}$ stand for the $j$ th and $n$th intrusion feature respectively.

Through the $k$ nearest neighbor graph method, the relationship among features is constructed. Furthermore, the adjacency matrix $\boldsymbol{A}$ which describes the relationship of intrusion features can be represented as

$$
\boldsymbol{A}_{i, j}= \begin{cases}1, & \text { if }\left(\boldsymbol{x}_{j}, \boldsymbol{x}_{n}\right) \in \mathscr{M} \\ 0, & \text { if }\left(\boldsymbol{x}_{j}, \boldsymbol{x}_{n}\right) \in \mathscr{N}\end{cases}
$$

where $x_{j}$ and $x_{n}$ stand for the $j$ th and $n$th intrusion feature.

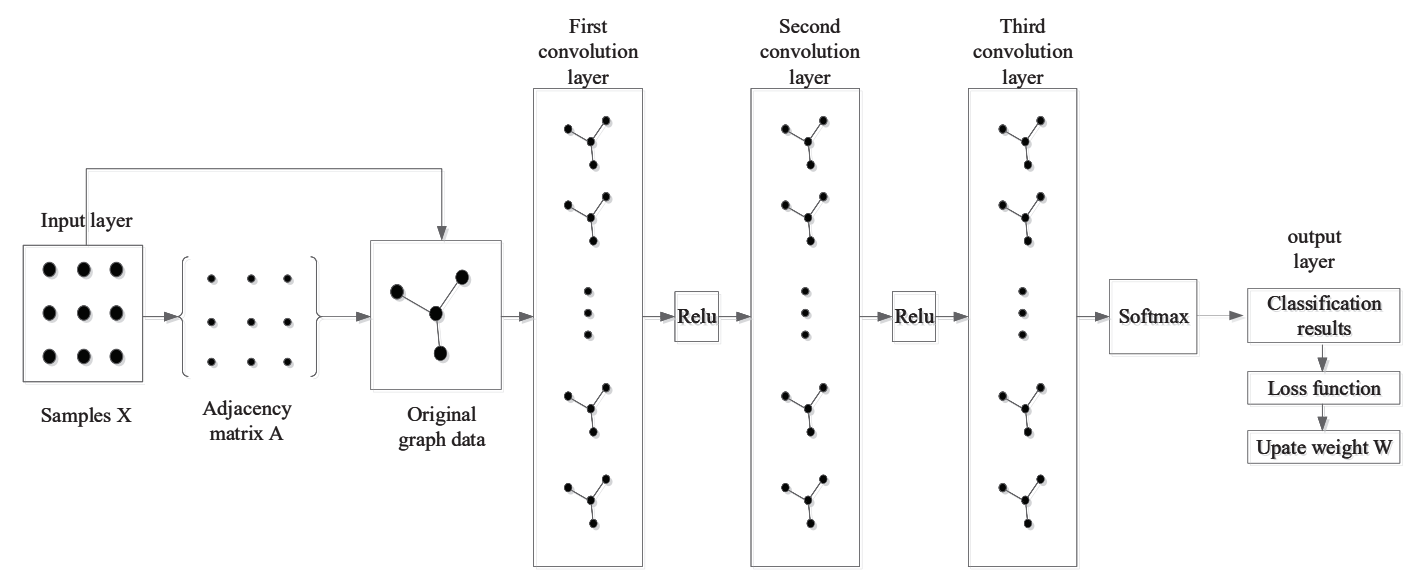

Fig. 2: Framework of a three-layer GCN model

The adjacency matrix $\boldsymbol{A}$ and the original intrusion data $\boldsymbol{X}$ are used as the input of GCN. Then the relationship between intrusion detection features can be further extracted through the graph convolution layer. The graph convolution layer is constructed based on a first order spectral graph convolution. The convolution operation on topological graph is realized by the theory of spectrum. The graph convolution can extract node and structural characteristics of the graph. Fig.2 shows a three-layer GCN model. Generally, the graph convolution layer in this paper can be expressed as

$$
\boldsymbol{H}^{l+1}=\operatorname{Relu}\left(\tilde{\boldsymbol{D}}^{-(1 / 2)} \tilde{\boldsymbol{A}} \tilde{\boldsymbol{D}}^{-(1 / 2)} \boldsymbol{H}^{(l)} \boldsymbol{W}^{(l)}\right)
$$


where $\tilde{\boldsymbol{A}}$ denotes a matrix with self-connection structure. $\tilde{\boldsymbol{D}}$ stands for the degree matrix with $\tilde{\boldsymbol{D}}_{i i}=\sum_{j} \tilde{\boldsymbol{A}}_{i j}$. $\boldsymbol{W}^{(l)}$ is the weight matrix of the specific layer. $\boldsymbol{H}^{(l)}$ means the feature information matrix in the $l$ th layer with $\boldsymbol{H}^{(0)}=\boldsymbol{X}$.

Generally, the network layer needs activation functions to improve the nonlinear representation ability. Relu function is easier to obtain the derivative and hence can accelerate network training. Therefore, Relu activation function is added between the graph convolution layers.

\subsection{Classifier Module}

Through the aforementioned feature extraction module, the attack features can be effectively extracted. Then the extracted features are put into the classifier module for classification. The Softmax classifier is a model with a polynomial distribution, which can be divided into multiple exclusive categories. The Softmax classifier used in this module is given by

$$
f\left(z_{i}\right)=\frac{e^{z_{i}}}{\sum_{j} e^{z_{j}}}
$$

where $z_{i}$ indicates the output value of the $k$ th neuron. $n$ is the intrusion detection number of categories.

The output of the Softmax layer is also a vector with the element value ranges from 0 to 1 . Each element in the vector is the probability of each class. The probability is used to express the relationship between the estimated value and the true value. Then the relationship can be measured by the cross entropy loss function in the back propagation of neural network model. The cross entropy loss function can be expressed as

$$
C=-\sum_{i} y_{i} \log f\left(Z_{i}\right)
$$

where $y_{i}$ represents the true network intrusion value corresponding.

Through the above three main modules, the intrusion detection model is established. The network parameters of the model can be further optimized until the network converges. In the above optimization procedure, the Adam optimization function is used to design different adaptive rate for different parameters.

\section{Experiments}

\subsection{Experiment Setting}

The computer configuration used in the experiment is as follows: CPU i7-7700@3.6HZ, 8 Gb of memory, 1 Tb SSD, installed Windows 7 operating system, using Keras 2.1.2 as a deep learning framework and Python 3.6 as the programming language.

\subsection{KDD-CUP 99 Dataset}

KDD-CUP 99 comes from DARPA intrusion detection assessment program. KDD-CUP 99 dataset consists of 4,900,000 samples, which contains 41 features and a label. In fact, only 10\% of the KDD-CUP 99 dataset is used to train. In order to evaluate the effectiveness of the model, especially for the unknown attack types, the corrected.gz dataset from KDD-CUP 99 is choice to test namely corrected.gz dataset.

There are 494,021 samples in the training data and 311,029 samples in the test data. Each sample is marked as normal or attack. Only 24 types of attacks appear in the training set and there are 38 types of attacks in the testing dataset. In addition, similar attacks are regarded as a single category, which leads to form four major attacks categories, namely, DOS, Probe, R2L and U2R. Details of the KDD-CUP 99 dataset are shown in Table 1.

Table 1: KDD-CUP 99 data details

\begin{tabular}{lccc}
\hline Data type & & Training set & Test set \\
\hline Normal & & 97278 & 60593 \\
\hline \multirow{2}{*}{ Attack } & DOS & 391458 & 229853 \\
& Probe & 4017 & 4166 \\
& R2L & 1126 & 5189 \\
\hline Total & U2R & 52 & 228 \\
\hline
\end{tabular}




\subsection{Evaluation Metrics}

In this paper, the labels are grouped into 5 categories (Normal, DOS, Probe, R2L and U2R) for training and testing. The proposed GCNID can be used to solve the multiple classification problem. Four main outcomes can be extracted from the test results, namely, true positive (TP), true negative (TN), false positive (FP), and false negative (FN). To evaluate the multiple classification tasks, TP predicts a positive class as a positive class. TN predicts a negative class as a negative class. FP predicts a negative class as a positive class. FN a positive class as a negative class.

Then five evaluation indexes (accuracy, precision, recall, F1-score and FAR) are given by the above four statistics. The list of the used evaluation indexes definition are as follows.

(i)Accuracy shows the true prediction rate for all test sets as $\gamma_{\mathrm{acc}}=\frac{\mathrm{TP}+\mathrm{TN}}{\mathrm{TP}+\mathrm{TN}+\mathrm{FP}+\mathrm{FN}}$.

(ii)Precision is the accuracy of the classifier, that is, the rate at which the attack is correctly marked from all samples classified as an attack from the test set as $\gamma_{\text {pre }}=\frac{\mathrm{TP}}{\mathrm{TP}+\mathrm{FP}}$.

(iii)Recall is the integrity of the classifier, that is, the correct labelled attack rate for all attack samples in the test set. It is also called true positive rate (TPR), detection rate (DR), or sensitivity as $\gamma_{\mathrm{rec}}=\frac{\mathrm{TP}}{\mathrm{TP}+\mathrm{FN}}$.

(iv)F1-Score can be viewed as the harmonic mean of the precision and recall indicators as $\gamma_{\mathrm{fsc}}=\frac{2 \times \gamma_{\mathrm{pre}} \times \gamma_{\mathrm{rec}}}{\gamma_{\mathrm{pre}}+\gamma_{\mathrm{rec}}}$.

(v)The FAR shows that all normal samples in the test set are misclassified as normal sample rates for any attack category as $\gamma_{\mathrm{far}}=\frac{\mathrm{FP}}{\mathrm{FP}+\mathrm{TN}}$.

\subsection{Experiment Results}

In this section, several simulation results are provided to illustrate the performance of the proposed GCNID algorithm. The $k$ nearest neighbors for the adjacency matrix $\boldsymbol{A}$ are evaluated in the first experiment. Besides, the GCN layers of the proposed GCNID are analyzed in the second experiment. The experiment results compared with DNN, LSTM, GRU, CNN and GCNID are given.

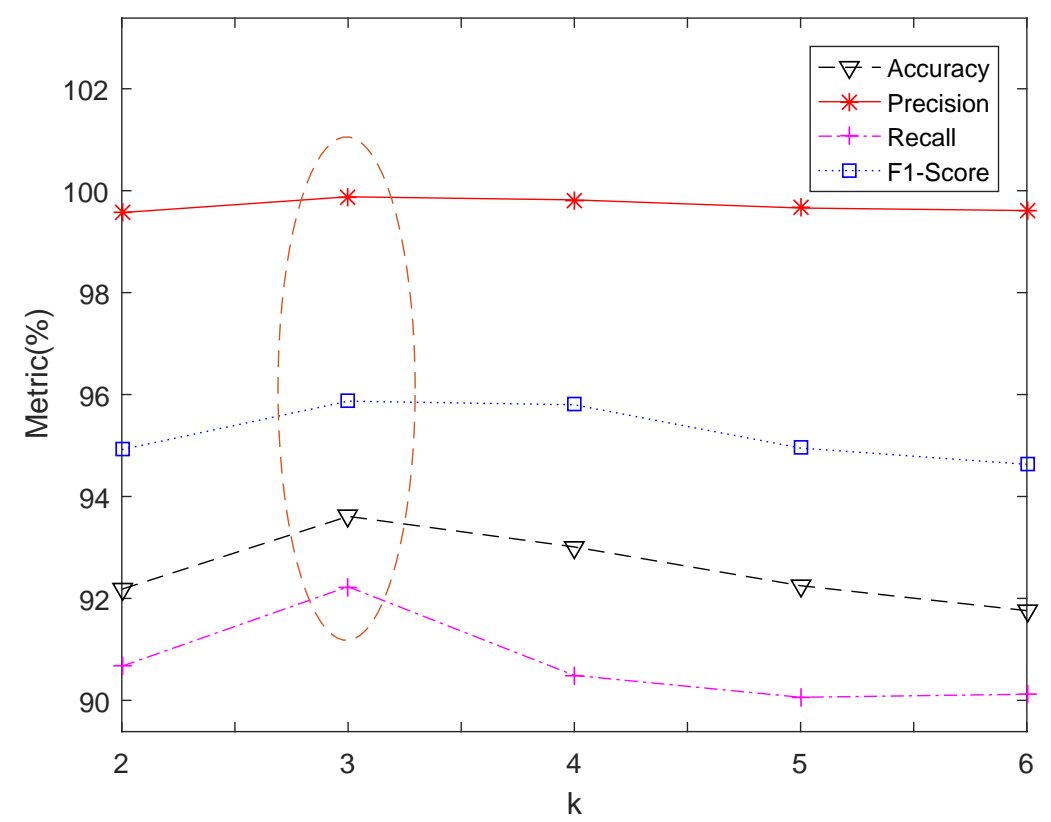

Fig. 3: Comparison of predicted performance versus different adjacency matrix

In the first experiment, Fig. 3 shows the experiment results of different $k$ nearest neighbors. Comparative experiment results are conducted for different $k$ values of the adjacency matrix constructed by $k$ nearest neighbor graph method. It is shown that the performances of intrusion detection are increased before $k=3$ and then the performance gets worse with the $k$ getting up. For intrusion detection, the results show that better experimental results can be obtained when $k=3$. Furthermore, $k=3$ is used for the following experiments.

Figure 4 shows experimental results of different GCN layers. As the increases of graph convolution neural network layer, the gradient of explosion or the disappearance of the gradient may happen. Respectively, the experiment 


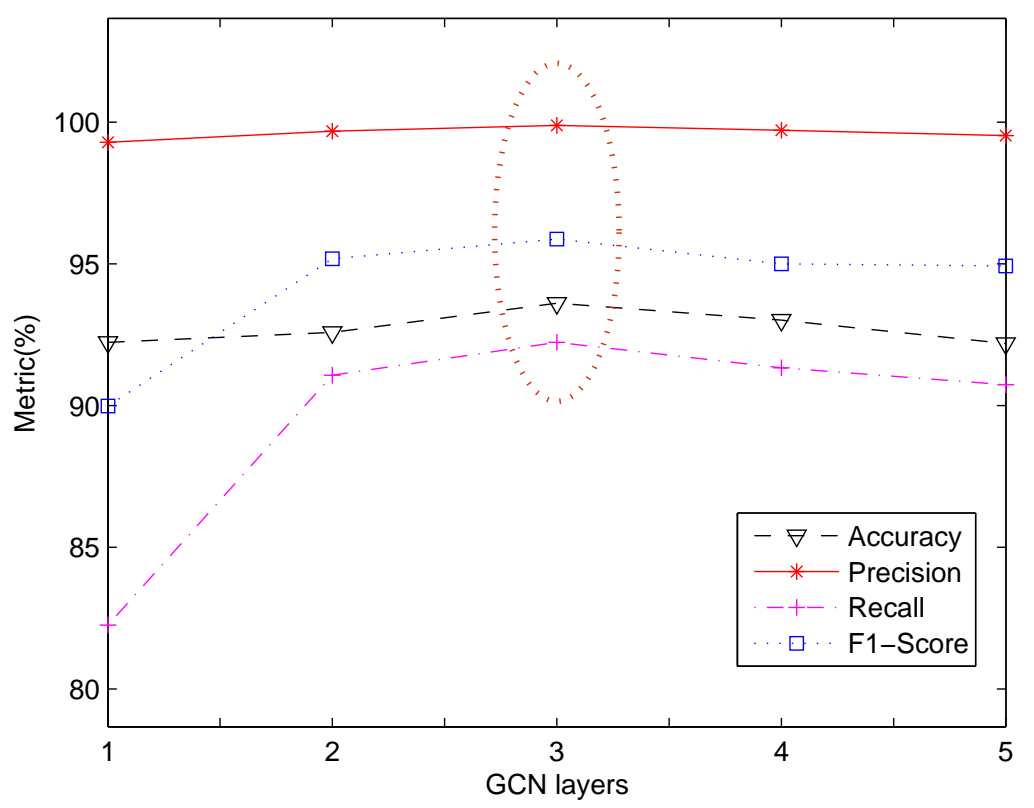

Fig. 4: Comparison of predicted performance versus different network layer

compared the number of $L=\{1,2,3,4,5\}$ GCN layers. It is shown that the performances of intrusion detection are increased before $L=3$ and then the performance gets worse with the $k$ getting up. The experimental results show that $L=3$ layer of GCN to the research of intrusion detection problem effect is best for the proposed GCNID. What's more, $L=3$ is used for the following experiments.

Table 2: KDD-CUP 99 experiment result

\begin{tabular}{lccccc}
\hline Metric & DNN $(\%)$ & LSTM $(\%)$ & GRU $(\%)$ & CNN $(\%)$ & GCNID $(\%)$ \\
\hline Accuracy & 90.77 & 92.17 & 91.98 & 93.07 & 99.62 \\
Precision & 95.19 & 99.58 & 99.85 & 91.24 & $\mathbf{9 3 . 6 1}$ \\
Recall & 90.12 & 90.66 & 90.17 & 95.24 & $\mathbf{9 2 . 2 3}$ \\
F1-Score & 93.63 & 94.91 & 94.76 & 2.11 & $\mathbf{9 5 . 8 7}$ \\
FAR & 5.86 & 1.58 & 1.72 & $\mathbf{1 . 2 6}$ \\
\hline
\end{tabular}

The experimental results are shown in Table 2 which compared with DNN, LSTM, GRU and CNN. Experimental results show that the model proposed in this paper obtains $93.61 \%$ accuracy. Compared with other methods, the proposed GCNID algorithm can effectively extract features of intrusion datas. Hence the proposed GCNID algorithm achieves a better performance and generalization ability for unknown attack types.

Figure 5 can intuitively compare the accuracy of different attacks with other methods. It is shown that the accuracy of the four type attacks by the proposed GCNID algorithm is higher than other methods, especially for the Probe and U2R. The recognition accuracy of DOS and R2L achieve minor lift compered with the other methods.

\section{Conclusion}

This paper presents an effective GCNID algorithm for multiple classification intrusion detection. The proposed algorithm utilizes the relation among the detection data to build a graph, and then can extract the structure and feature information of the network intrusion data via GCN. Simulation results show that the GCNID algorithm can not only improve the accuracy, precision, recall of the intrusion detection classification, but also have good generalization ability to detect unknown attack types. 


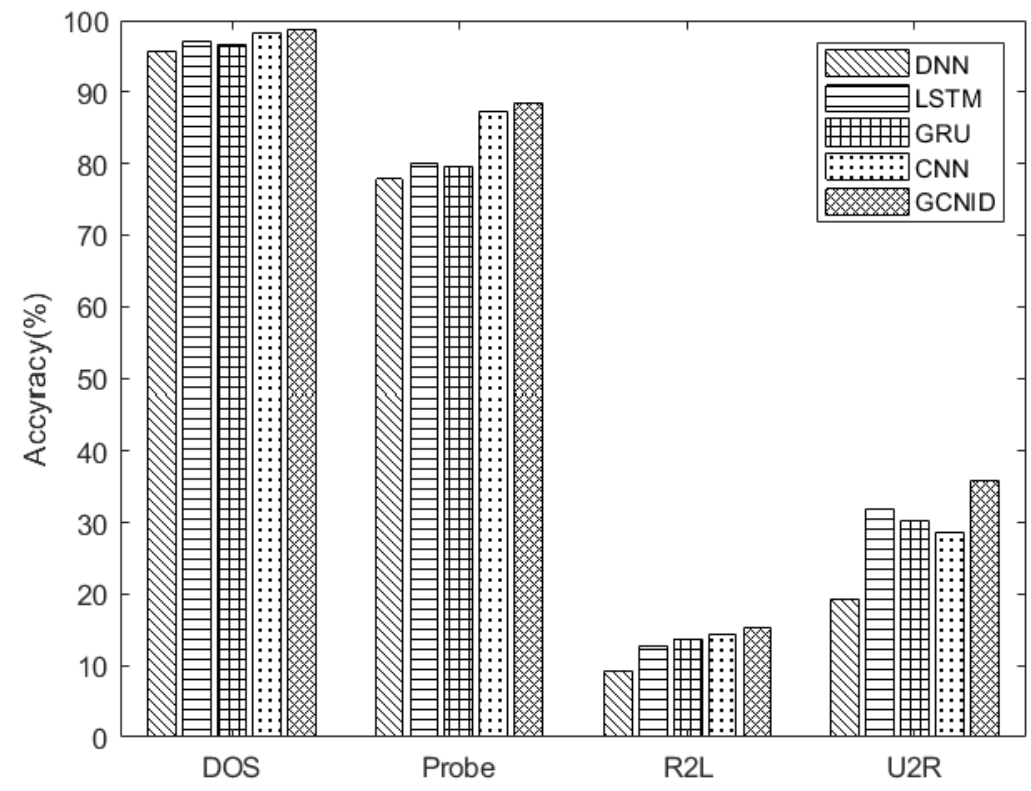

Fig. 5: Detection rate for each class in the dataset

\section{Conflicts of Interest}

The authors declare that there are no conflicts of interest regarding the publication of this paper.

\section{Acknowledgements}

This work was supported by the National Natural Science Foundation of China under Grant No.61971117, by the Natural Science Foundation of Hebei Province (Grant No. F2020501007).

\section{References}

1. Elmasry W , Akbulut A , Zaim A H. "Empirical study on multiclass classification-based network intrusion detection". Computational Intelligence,vol. 35, no. 4, pp. 919-954, NOV, 2019.

2. Shah V, Aggarwal AK,Chaubey N, Zaim A H. "Performance improvement of intrusion detection with fusion of multiple sensors". Complex \& Intelligent Systems, vol. 3, no. 1, pp. 33-39, MAR, 2017

3. Tang TA, Mhamdi L, McLernon D, Zaidi SAR, Ghogho M. "Deep learning approach for network intrusion detection in software defined networking".Electronics, vol. 9, no. 9, SEP 2020.

4. Feng T, Dou MF.'A weighted intrusion detection model of dynamic selection". Applied Intelligence JAN 2021

5. Ramaki AA, Amini M, Atani RE. "RTECA: Real time episode correlation algorithm for multi-step attack scenarios detection". Computational Intelligence,vol. 49, pp. 206-219, MAR 2015.

6. Feng T, Dou MF."A weighted intrusion detection model of dynamic selection". Applied Intelligence JAN 2021

7. Xu X, Lima S, Rocha, alvaro. "Simulation analysis of intrusion detection system based on genetic attribute reduction algorithm and neural network based on rough set theory".Journal of Intelligent \& Fuzzy Systems.vol. 35, no. 3, pp. 2937-2942, 2018

8. Liu Y, Zhu L. "A new intrusion detection and alarm correlation technology based on neural network".EURASIP Journal on Wireless Communications and Networking.vol. 35, no. 3, MAY 2019

9. Hinton G,Deng L, Yu D, Dahl GE, Kingsbury B. ”Deep Neural Networks for Acoustic Modeling in Speech Recognition: The Shared Views of Four Research Groups".IEEE Signal Processing Magazine.vol. 29, no. 6, pp. 82-97, NOV 2012

10. Jia Y, Wang Meng, Yagang W. "Network intrusion detection algorithm based on deep neural network".IET information security.vol. 13, no. 1, pp. 48-53, JAN 2019

11. Kim Jihyun, Kim Jaehyun. 'Long Short Term Memory Recurrent Neural Network Classifier for Intrusion Detection”.3rd International Conference on Platform Technology and Service (PlatCon) Location: ICT Platform Soc, SOUTH KOREA. 2016

12. Ding S, Wang G. "Research on intrusion detection technology based on deep learning".IEEE 2017 3rd IEEE International Conference on Computer and Communications (ICCC).pp. 1474-1478, 2016

13. Liu G , Zhang J . "CNID: Research of Network Intrusion Detection Based on Convolutional Neural Network". Discrete Dynamics in Nature and Society. MAY 2020.

14. Kipf, T.N., Welling, M. "Semi-supervised classification with graph convolutional networks". Conf. Learning Representations, Toulon, France, April 2017.

15. Fu S, Liu W, Li S, Zhou Y. "Two-order graph convolutional networks for semi-supervised classification”.IET Image Processing.vol. 13, no. 14, pp. 2763-2771, DEC 122019 
16. D. Kingma and J. Ba. "ADAM: a method for stochastic optimization". https://arxiv.org/abs/1412.6980v9,(2017)

17. Zhan M, Gan J, Lu G. "Graph convolutional networks of reconstructed graph structure with constrained Laplacian rank".Multimedia Tools and Applications. OCT 2020

18. Boulaiche, Ammar, Adi. "An auto-learning approach for network intrusion detection".Telecommunication Systems Modeling Analysis Design \& Management.vol. 68, no. 2, pp. 277-294, JUN 2018 
Figures

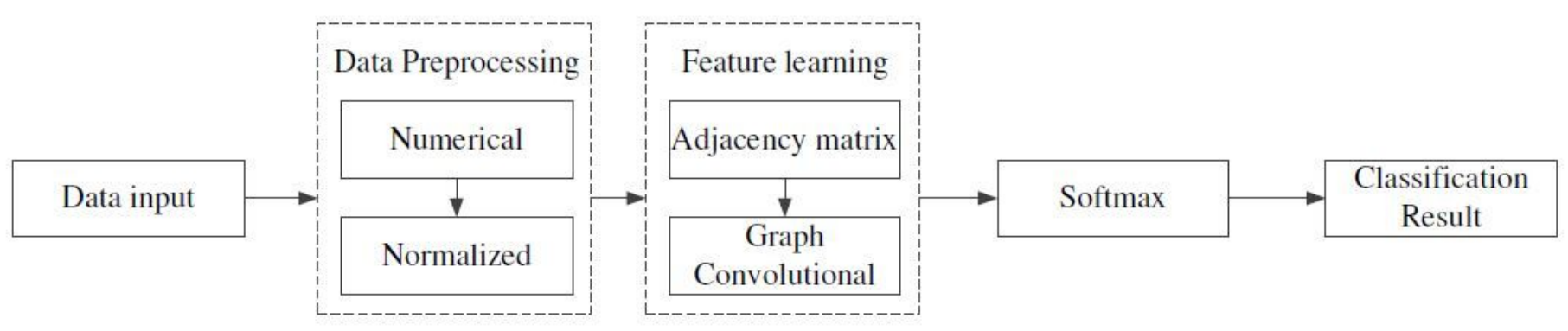

Figure 1

GCN-based network instruction detection architecture
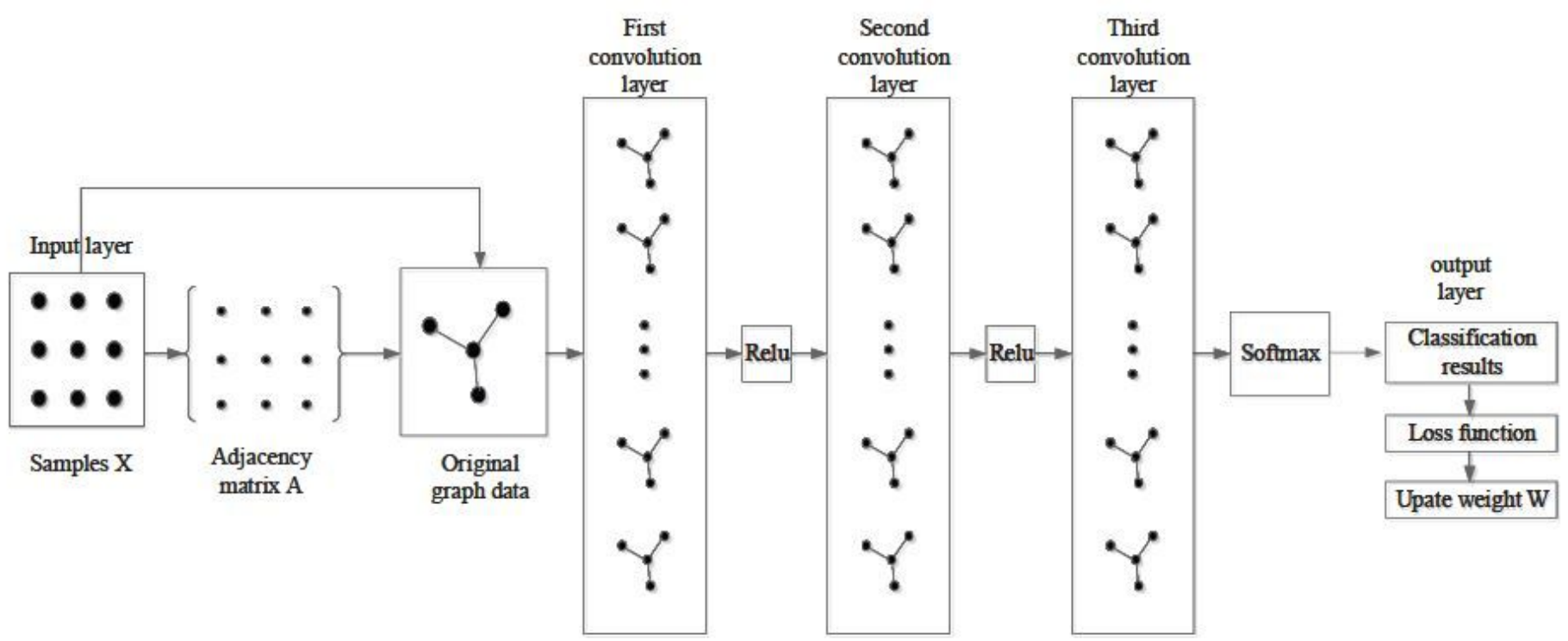

Figure 2

Framework of a three-layer GCN model 


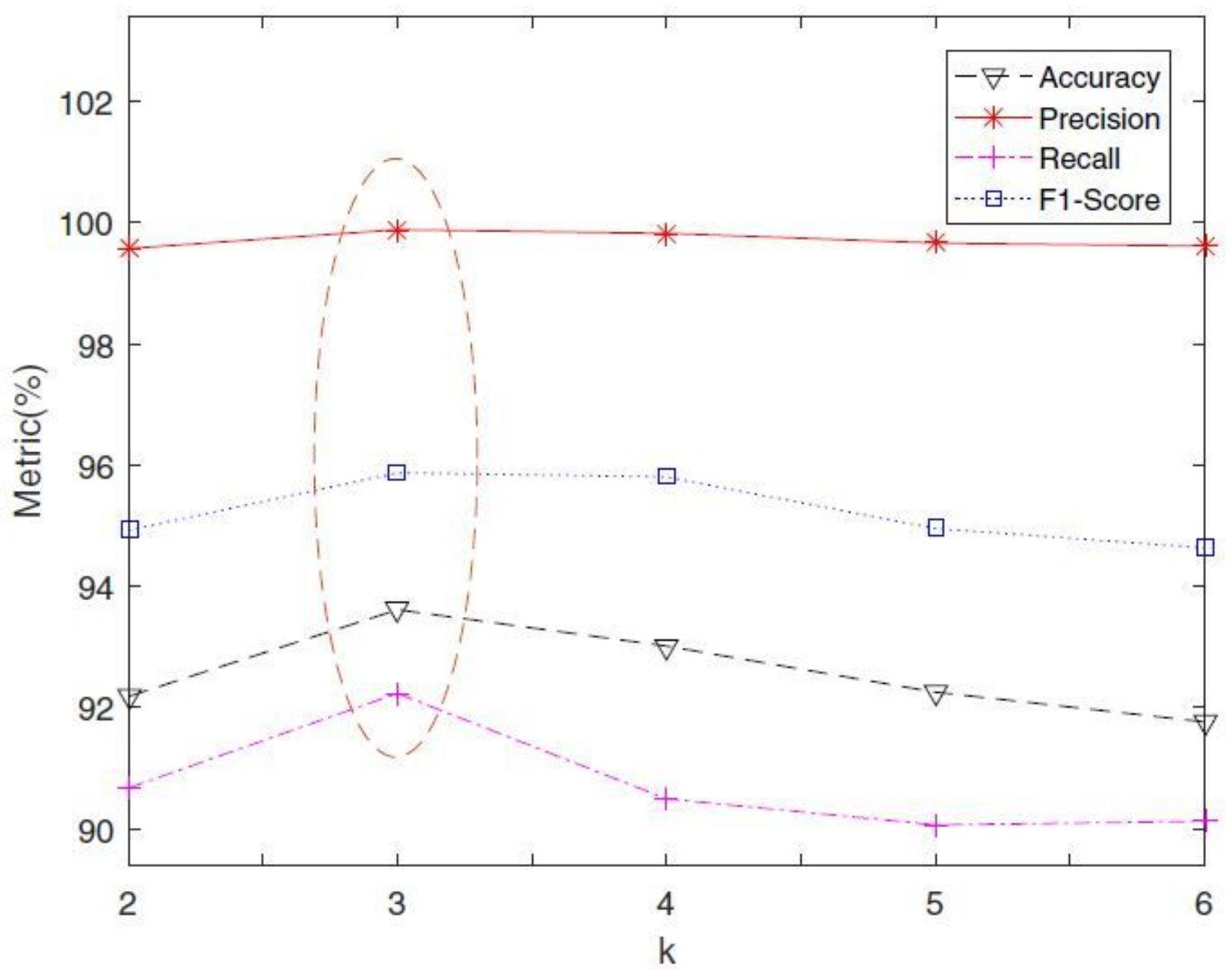

Figure 3

Comparison of predicted performance versus different adjacency matrix 


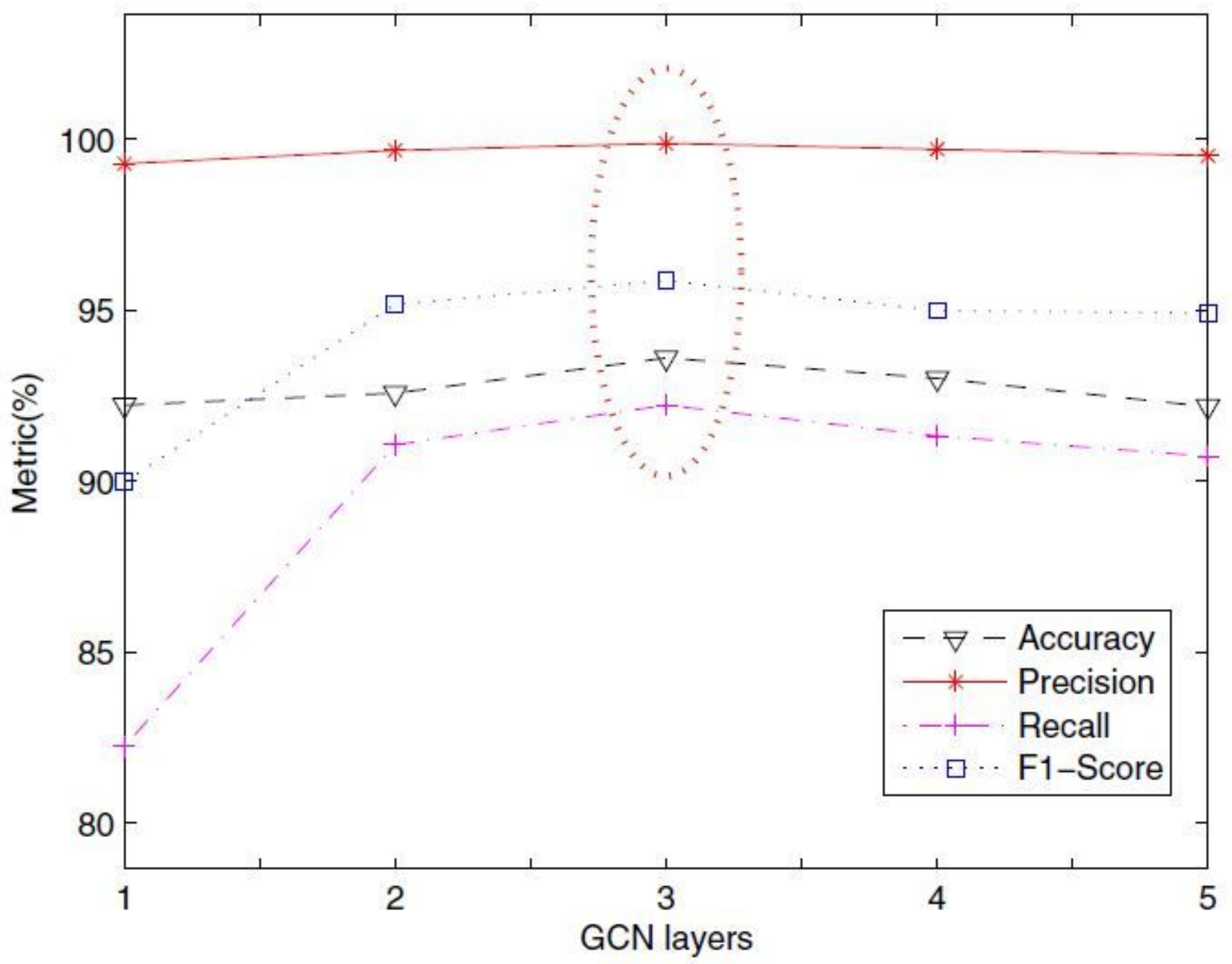

Figure 4

Comparison of predicted performance versus different network layer 


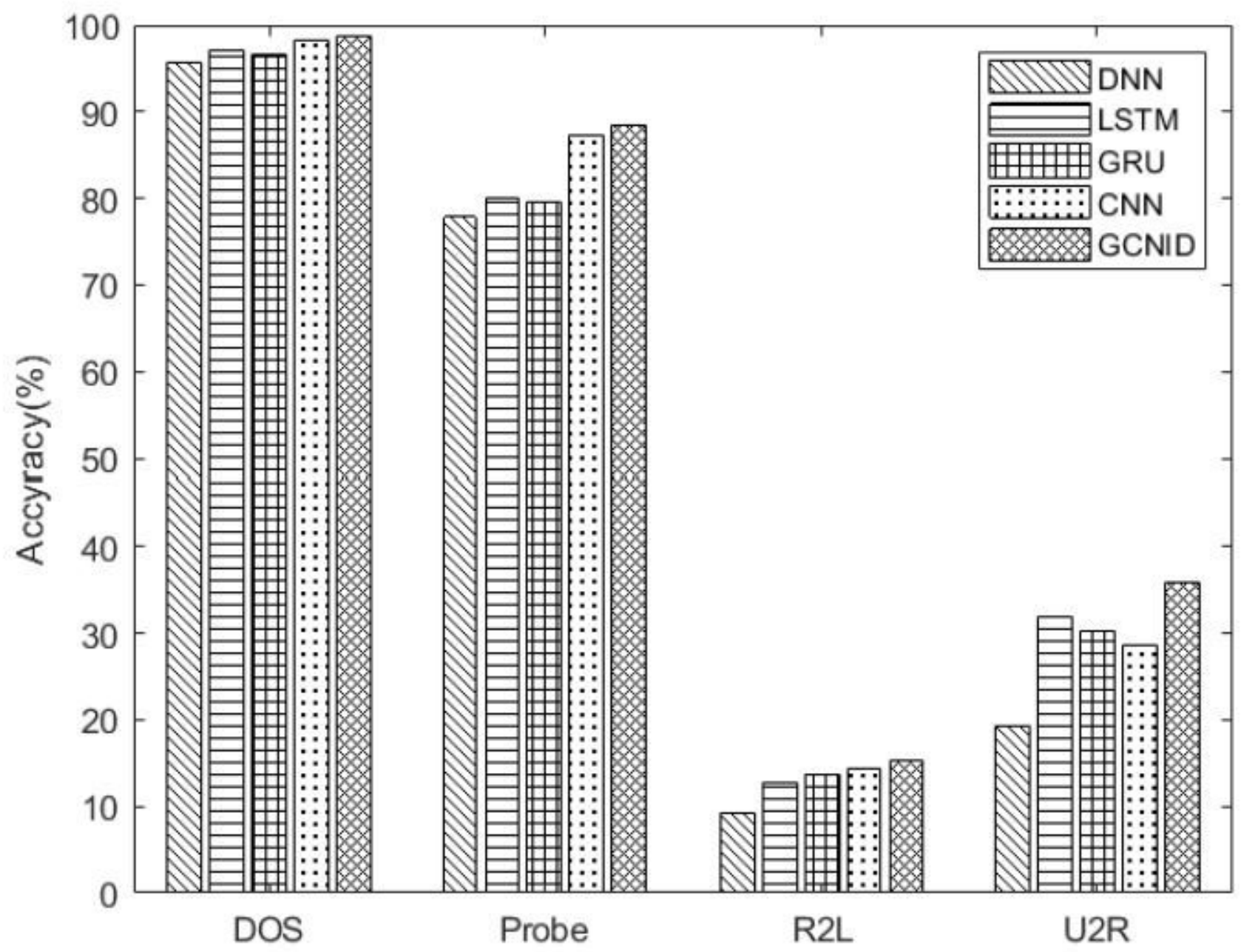

Figure 5

Detection rate for each class in the dataset

\section{Supplementary Files}

This is a list of supplementary files associated with this preprint. Click to download.

- latex.rar 